\title{
Chronic Care Management: Bahn frei für ein neues Betreuungsmodell
}

\author{
Was 2009 als Gedankenskizze startete, erreichte 2011 ein denkwürdiges Zwischen- \\ ziel: Sieben Ärzteorganisationen und fünf Versicherer einigten sich auf eine Pauschal- \\ vergütung für ärztliche und nichtärztliche Betreuungsleistungen beim Futuro Chronic \\ Care Management. Das neue Versorgungsmodell erweitert die hausärztlichen Kapa- \\ zitäten mit speziell weitergebildeten Praxisassistentinnen.
}

Urs Zanoni

Projektleiter Futuro Chronic Care Management

Korrespondenz: Urs Zanoni, MPH Giessenweg 3 CH-5022 Rombach
Am Anfang standen Erkenntnisse, die allgemein bekannt waren, doch es fehlten Lösungen:

- Die Zukunft ist chronisch: Gemäss WHO werden 2020 mindestens 60 Prozent aller Erkrankungen chronischer Natur sein.

- Es mangelt an hausärztlichen Kapazitäten: Das Schweizerische Gesundheitsobservatorium geht davon aus, dass sich die Lücke zwischen nachgefragten und angebotenen Konsultationen beim Hausarzt von 2010 bis 2030 um eine halbe Million pro Jahr vergrössert.

- Die Betreuung von chronisch kranken Patienten ist lückenhaft: Untersuchungen im In- und Ausland zeigen, dass chronisch Kranke häufig nicht die Versorgung erhalten, die sie benötigen.

Folglich lautete die Schlüsselfrage: Wie lässt sich die Betreuung von multimorbiden, chronisch kranken Patienten verbessern, obwohl die hausärztlichen Ressourcen knapp sind? Die Antwort heisst Futuro Chronic Care Management (Futuro CCM): ein hausarztbasiertes Betreuungsmodell mit spezifisch weitergebildeten Medizinischen Praxisassistentinnen (MPA) und intelligenten Arbeitsinstrumenten, ausgehend vom Chronic Care Model und weiteren Best-PracticeKonzepten.

Im Vordergrund steht die Qualität der Betreuung: Die Kandidaten für das Futuro CCM werden proaktiv angesprochen und angeschrieben. Die umfassende Betreuung schliesst auch den datenschutzkonformen Austausch zwischen allen Beteiligten ein. Die regelmässige Auswertung der erhobenen Daten ermöglicht es, die Prozesse laufend zu überprüfen und gegebenenfalls anzupassen. Damit erhalten die Patienten die bestmögliche Betreuungsqualität und Versorgungssicherheit.

Angeschoben wurde das Projekt von mediX bern und mediX zürich. Inzwischen bilden sieben Ärzteund Praxisorganisationen den Projektausschuss und entwickeln das Vorhaben Schritt für Schritt weiter: eastcare, LuMed, Meconex, mediX bern, mediX zürich, Sanacare, Swica Gesundheitszentren. Sie vertreten

\section{Chronic Care Management:} champ libre pour un nouveau modèle de soins

L'idée lancée en février 2009 a fait son chemin: en septembre 2011, sept organisations de médecins et cinq assureurs se sont mis d'accord sur une rémunération forfaitaire pour les prestations médicales et non-médicales de suivi dans le cadre du projet «Futuro Chronic Care Management». Le nouveau modèle de soins élargit les capacités du médecin de famille grâce à des assistantes médicales spécialement formées. Les formations destinées aux assistantes démarreront début 2012, de sorte que les premiers patients «Futuro» puissent être pris en charge début 2013.

gut 1000 Grundversorger und schaffen damit eine starke Basis für die Verbreitung des Modells.

Ein zentrales Element im Futuro CCM ist die MPA. Sie wird spezifisch weitergebildet und arbeitet im Auftrag und unter Aufsicht des Arztes. Bei Bedarf wird das Betreuungsteam durch weitere Leistungserbringer wie Spezialisten, stationäre Einrichtungen, Spitex, Fachorganisationen oder Apotheker ergänzt.

Die Weiterbildung der MPA umfasst in einer ersten Phase zwei Basismodule zum Chronic Care Management. Diese Module befähigen sie, spezifische Betreuungsleistungen zu übernehmen:

- Zum Auftakt ein Round-Table-Gespräch mit dem Patienten und dem Arzt, in dem die Ausgangslage erfasst und die Betreuung geplant wird (Status der Diagnosen und Medikation, Zielvereinbarungen, Bestimmung der Messgrössen, Wahl der Schulungsmodule). 
- Persönliche Beratungen des Patienten in der Hausarzt-Praxis (Grundlagen: strukturierter Fragebogen, Zielvereinbarungen, Meldungen des Patienten zum Verlauf; Themen: Alarmsymptome, Medikamente, Gesundheitsverhalten, Alltagsstruktur und Sozialkontakte, Umgang mit Rückschlägen).

- Patientenschulungen (in Ergänzung zum Arzt und anderen Fachpersonen).

- Telefonisches Coaching des Patienten (Grundlagen und Themen analog zur Beratung in der Praxis).

- Monitoring des Patienten und Interventionen nach Absprache mit dem Arzt (Grundlagen: erfasste Messwerte, Verlaufsmeldungen des Patienten, Zielvereinbarungen).

In einer zweiten Phase wird die Weiterbildung durch krankheitsspezifische Module ergänzt. Die Inhalte der beiden Basismodule wurden in Absprache mit dem Berufsverband der Praxisassistentinnen (SVA), der OdA Berufsbildung MPA, dem Institut für Hausarztmedizin der Universität Zürich sowie dem Weiterbildungszentrum für Gesundheitsberufe in Aarau entwickelt. Die beiden Module werden im Frühjahr 2012 erstmals angeboten. Die Ausschreibung ist auf der Website www.careum-weiterbildung.ch $(\rightarrow$ Lehrgänge) zu finden.

Den Projektträgern war von Beginn weg klar, dass sich das Futuro CCM nur dann durchsetzen wird, wenn die Betreuungsleistungen der MPA vergütet sind. Deshalb war die FMH bereit, einen TARMEDAntrag für ein neues Unterkapitel «Chronic Care Management» mit vier ärztlichen und vier nichtärztlichen Positionen einzureichen. Der Antrag liegt seit Januar 2011 bei der Paritätischen Tarifkommission.

Parallel dazu fanden Gespräche mit Versicherern statt über die Zahlung von Betreuungspauschalen während einer dreijährigen Pilotphase (2013-2015). Bis Ende September genehmigten fünf Versicherer einen Vorvertrag, der die vergüteten Leistungen und die Höhe der Pauschale nennt: atupri, Concordia, Sympany, Swica, Visana. Damit diese «Allianz der Motivierten» den Vertrag definitiv in Kraft setzt, müssen bis September 2012 die letzten Rahmenbedingungen einvernehmlich geklärt sein, namentlich das Qualitätsmanagement und der Datenaustausch.

Mit diesem Vorvertrag und der MPA-Weiterbildung steht das Futuro CCM auf einem tragfähigen Fundament. Ebenso tragfähig ist die Allianz der Motivierten, sowohl aufseiten der Leistungserbringer wie aufseiten der Versicherer.

Die Projektträger sind überzeugt, dass das Futuro CCM allen Beteiligten einen messbaren Mehrnutzen bringt:

- Patienten: Sie können in ihrer vertrauten Umgebung kompetent und umfassend betreut werden. Dadurch steigt ihre Lebensqualität. Dank der erlernten Handlungskompetenzen sind sie selb- ständiger. Bei Bedarf werden Angehörige oder andere Vertrauenspersonen in die Betreuung einbezogen.

- Arzt: Er wird von zeitintensiven Arbeiten entlastet (Information, Schulung, Instruktion) und schafft Kapazität für anspruchsvolle diagnostische und therapeutische Leistungen, die seine besonderen Fähigkeiten erfordern. Die Betreuungsleistungen, die er an die MPA delegiert, sind angemessen vergütet.

- MPA: Sie übernimmt neue Aufgaben und Verantwortlichkeiten, was das Selbstbewusstsein und die Leistungsbereitschaft stärkt. Das Erlernte erlaubt ihr, gezielter und bewusster auf die Bedürfnisse der Patienten einzugehen. Damit trägt sie massgeblich zu besseren Betreuungsergebnissen bei.

- Versicherer: Das Futuro CCM fokussiert auf eine kostenintensive Patientengruppe und motivierte Leistungserbringer (der Arzt investiert, nicht ein Dritter). Das Risiko ist überblickbar, da der Einschluss der Patienten eine Kostengutsprache verlangt. Zudem ist das Betreuungsmodell evidenzbasiert, und die dreijährige Pilotphase wird wissenschaftlich begleitet.

Bisher wird das Projekt von den genannten Ärzteund Praxisorganisationen getragen. Nun ist ein Verein geplant, in dem Leistungserbringer und Versicherer gleichberechtigt vertreten sind. Dieser FuturoVerein legt den Rahmen fest, in dem das Betreuungsmodell umgesetzt wird. Dazu gehören zum Beispiel: Muss-Kriterien für die Behandlungsempfehlungen, die Anforderungen an die Arbeitsinstrumente von Arzt und MPA, das Qualitätskonzept (inkl. Qualitätsindikatoren), das Konzept für das Datenmanagement, die Spezifikation von Schnittstellen und Protokollen für den Datenaustausch.

Innerhalb dieses Rahmens setzen die Leistungserbringer das Futuro CCM eigenverantwortlich um. Voraussetzung dafür ist ein Attest, vergleichbar der ärztlichen Dignität. Wer das Attest erhalten möchte, muss sich verpflichten, bei der Umsetzung die Vorgaben des Futuro Vereins einzuhalten. Praxen mit dem Futuro Attest können die vereinbarten Betreuungspauschalen abrechnen. Das Attest wird regelmässig überprüft und erneuert.

Für die Umsetzung werden den Praxen Produkte und Dienstleistungen angeboten. Ob dafür eine eigenständige, vom Verein unabhängige Firma gegründet wird, ist offen. Die Alternative sind bestehende Anbieter, die solche Produkte und Dienstleistungen aufgrund der Vergaben des Vereins - entwickeln und den Praxen offerieren.

Anfang 2012 soll der geplante Futuro Verein gegründet werden. Die MPA-Weiterbildungen starten im Frühjahr 2012, sodass Anfang 2013 die ersten Futuro Patienten betreut werden können. 